DE

M E D I C I N A

T R O P I C A L

DE

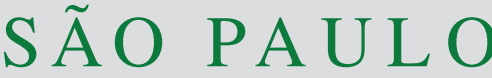

JOURNAL OF THE SÃO PAULO INSTITUTE OF TROPICAL MEDICINE

${ }^{1}$ Fundação Oswaldo Cruz, Instituto Nacional de Infectologia Evandro Chagas, Laboratório de Epidemiologia Clínica, Rio de Janeiro, Rio de Janeiro, Brazil

${ }^{2}$ Fundação Oswaldo Cruz, Instituto Fernandes Figueira, Departamento de Pediatria, Rio de Janeiro, Rio de Janeiro, Brazil

${ }^{3}$ Fundação Oswaldo Cruz, Instituto Fernandes Figueira, Departmento de Doenças Infecciosas, Rio de Janeiro, Rio de Janeiro, Brazil

${ }^{4}$ Universidade Estácio de Sá, Rio de Janeiro, Rio de Janeiro, Brazil

${ }^{5}$ Escola de Medicina Souza Marques, Rio de Janeiro, Rio de Janeiro, Brazil

Correspondence to: Dulce Helena Gonçalves Orofino

Fundação Oswaldo Cruz, Instituto Fernandes Figueira, Departamento de Pediatria, Av. Rui Barbosa, 716, CEP 22250-020, Rio de Janeiro, RJ, Brazil Tel: +5521986292498

E-mail: dulceorofino@yahoo.com.br

Received: 25 March 2020

Accepted: 30 June 2020

\section{4-hour Holter findings in infants with in-utero exposure to the Zika virus: a series of cases}

Dulce Helena Gonçalves Orofino ${ }^{(1,2,5}$, Sonia Regina Lambert Passos ${ }^{(1)}$ 1,4, Sheila Moura Pone ${ }^{\circledR 3}$, Marcos Vinícius da Silva Pone ${ }^{\circledR 3}$, Elisa Barroso de Aguiar $^{\left({ }^{1} 3\right.}$, Igo Oliveira de Araújo ${ }^{\circledR 3}$, Thiago Moreira Ramos ${ }^{\circledR} 4$, Letícia Machado Lima e Silva ${ }^{(1)}$, Bruna Menezes de Oliveira ${ }^{(1)}$, Luan Noé da Silva ${ }^{\circledR 1}$, Rafaela Valentim Goldenzon ${ }^{\circledR 5}$, Maria Elizabeth Moreira ${ }^{\circledR 2}$, Raquel de Vasconcellos Carvalhaes de Oliveira ${ }^{\circledR 1}$

\section{ABSTRACT}

Severe neurological problems and other special manifestations such as high prevalence of structural cardiac changes has been described in infants vertically exposed to the Zika virus (ZIKV) and has been called congenital Zika virus syndrome (CZS). Previous studies have shown that the 24-hour Holter heart rate variability (HRV) analysis allows the prediction of worse outcomes in infants with neurological impairment and higher risk of sudden infant death syndrome (SIDS), hypertension, diabetes mellitus and other cardiovascular diseases. This study describes the 24-hour Holter findings of infants with confirmed vertical exposure to the ZIKV by positive polymerase chain reaction (PCR) assays in the mother's blood during pregnancy and/or in the urine or cerebrospinal fluid of the newborn. Data analysis was descriptive and included two subgroups according to the presence of fetal distress, positive PCR to ZIKV in the newborn, CZS and severe microcephaly. Heart rate, pauses, arrhythmias, ST segment and QT interval analyses and HRV evaluation through R-R, SDNN, pNN50 and rMMSD were described. The Mann-Whitney test was performed to assess differences between the two subgroups. The sample consisted of 15 infants with a mean age of 16 months, nine of whom were male. No arrhythmias or QT interval changes were observed. The comparison of HRV through the Mann-Whitney test showed a significant difference between patients with and without CZS, with and without severe microcephaly, with lower HRV in the groups with severe microcephaly and CZS. The study suggests that there is an increased risk of SIDS and cardiovascular diseases in this group of patients.

KEYWORDS: Zika virus. Holter. Arrhythmias. Heart rate variability. Congenital Zika Virus Syndrome. Congenital infection. Vertical transmission.

\section{INTRODUCTION}

The autonomic nervous system (ANS) and the balance of its sympathetic and parasympathetic pathways regulates the cardiovascular function during the intrauterine life ${ }^{1}$. Studies associate the imbalance between the sympathetic and parasympathetic ANS with the development of hypertension, cardiovascular disease, diabetes mellitus and asthma, as well as cognitive and behavioral changes ${ }^{2}$.

The knowledge of the anatomy and the development of the ANS have allowed a better understanding of the ANS involvement in cardiac arrhythmias. There are reports in the literature suggesting that the ANS dysregulation caused by gene 
polymorphisms that modulate the ANS development may be implicated in sudden infant death syndrome (SIDS). Furthermore, an imbalance between the sympathetic and parasympathetic ANS can lead to cardiac arrhythmias, and the predominance of one over the other may vary depending on the type of arrhythmia. For example, in the Brugada syndrome, arrhythmic events have been associated with periods of higher parasympathetic tone. In catecholaminergic polymorphic ventricular tachycardia, ventricular arrhythmia events are associated with the predominance of the sympathetic ANS $^{3}$.

The gold standard for studying the electrical activity of the heart is the 24-hour Holter monitoring, which records the electrical activity of the patient in his or her usual routine for 24 hours 4 . Fluctuations in the heart rate (HR) are related to neural activity, and HR variability (HRV) that is obtained through the 24-hour Holter monitoring is the end result of the integrated and rhythmic activity of ANS neurons generated by organized cardiorespiratory reflexes being recognized as a sign of heart health ${ }^{5,6}$. HRV assessment is performed using time domain measures, such as mean $R-R$ values (measured between 2 consecutive $R$ waves of the trace), SDNN (standard deviation of R-R intervals throughout the Holter "clean trace" time), pNN50 (the mean number of times per hour when the R-R intervals exceed 50 milliseconds) and rMMSD (the mean square root of successive differences between normal heart beats) and by frequency domain measurements (low, high and very high) after the software analyzing the exam has deleted the trace periods with interference. The heart rate increases rapidly in the first week of life and then decreases, and HRV decreases in this early neonatal period (following the highest HR) and then increases, indicating a predominance of the parasympathetic ANS over the sympathetic ANS after six months of age ${ }^{5,7}$. Studies conducted since the 1980s have suggested that HRV assessment may be a useful tool to provide information about neurodevelopment in children exposed to risks of perinatal brain damage because HRV would be related not only to the functional integrity of the ANS but also to the entire central nervous system $(\mathrm{CNS})^{8,9}$.

Recently, the introduction of the Asian lineage of ZIKV in 2015-2016 in Brazil was accompanied by severe damage to the CNS and other organs of fetuses exposed to ZIKV in the intrauterine period. CZS is defined as the combination of microcephaly, specific changes in CNS imaging (computed tomography), ophthalmic alterations and arthrogryposis ${ }^{10}$. Fetal ultrasound findings such as microcephaly were reported in $82.3 \%$ of the cases, and newborn skull tomography findings such as ventriculomegaly (93.8\%), cerebral calcifications $(98.8 \%)$, and poorly developed cerebellum or cerebellar vermis $(51.8 \%)$ were reported ${ }^{11}$.
Although not part of the CZS, recent studies have shown a higher prevalence of structural alterations in newborns and infants with confirmed vertical exposure to ZIKV by twodimensional Doppler echocardiograms than in the general population of live births ${ }^{12}$, however, there are no data in the literature on the changes in the electrical activity of the heart of these children. In view of these findings and the importance of $\mathrm{HRV}$ in assessing the integrity of the nervous system, the 24-hour Holter monitoring is justified in infants with confirmed vertical exposure to ZIKV due to a potential CNS impairment in these patients.

\section{MATERIALS AND METHODS}

The study was approved by the Research Ethics Committee of the National Institute of Infectious Disease (INI-FIOCRUZ - CAAE No 62728516.2.0000.5262), and all the caretakers agreed to sign the informed consent form. Fifteen patients were included in the cohort study of newborns and infants with vertical exposure to ZIKV, held from November 2015 to date, at the Instituto Fernandes Figueira (IFF), Rio de Janeiro, Brazil. All the infants had negative serologyical result for other congenital infections including toxoplasmosis, rubella, cytomegalovirus, hepatitis B, C and HIV, in addition to the exclusion of genetic syndromes potentially accompanied by congenital defects. The Holter exams were performed when the patients were hospitalized for clinical or surgical reasons, on the day before hospital discharge. Vertical exposure was confirmed by a positive polymerase chain reaction (PCR) amplification to ZIKV from the mothers' blood samples during pregnancy and/or in the newborn urine or cerebrospinal fluid soon after birth ${ }^{13,14}$.

The sample was divided into subgroups, according to the following characteristics: sex (female or male); positive or negative PCR to ZIKV in the newborn; with or without a history of fetal distress (Apgar score less than 6 in the $5^{\text {th }}$ minute of life) ${ }^{15}$; preterm (PT) (gestational age less than 36 weeks and 6 days) or not $\mathrm{PT}^{16}$; small for gestational age (SGA) or not SGA (according to the definition established in the literature ${ }^{17}$; microcephaly [head perimeter (HP) less than 2 standard deviations (SD) for age, sex and gestational age], severe microcephaly (HP less than 3 SD for age, sex and gestational age) or no microcephaly ${ }^{15}$; and with or without $\mathrm{CZS}^{8}$.

The patients' companions were instructed to fill out an activity record during the 24 hours, however, as most patients had CNS involvement, there were no reports of physical activity (walking, running) in 14 of the 15 patients in the sample (only 1 patient had no impairment of motor functions); therefore, 14 patients remained in their beds throughout the examination period. 
Using a DMS 300 device, Satellite Resting v.78 model and analysis of 3 leads, the continuous monitoring was performed for 24 hours with the patient in his or her usual routine. According to the guidelines of the Task Force of the European Society of Cardiology and the North American Society of Pacing and Electrophysiology ${ }^{18}$, only tests with more than 18 hours of "clean trace" were analyzed. To determine HRV parameters, a Holter software was used, and all the traces were subsequently analyzed individually by two cardiologists who were specialized in Holter analyses. The complexes classified as "noise" or with artifacts were manually excluded. Maximum, minimum and mean HR, pauses, presence of supraventricular and ventricular extrasystoles, bigeminal or trigeminal events, ST segment and QT changes were evaluated. The HRV assessment was performed through 24-hour R-R and SDNN, pNN50 and rMMSD. A nonparametric Mann-Whitney test was used to evaluate differences in median values among the groups of patients, using $p$-values $<0.10$ as suggestive of a significant difference.

Patients who presented with some alteration in the 24-hour Holter assessment were referred to the Cardiopediatrics outpatient clinic.

\section{RESULTS}

The sample consisted of 15 patients ( 9 males) with a mean age of 16 months. Two patients had a history of fetal distress, 4 patients were born SGA, 1 patient was born PT, and 12 had been diagnosed with CZS, and among these, 8 had severe microcephaly. From the total of 15 patients in the sample, 4 were hospitalized to undergo the Holter monitoring, and 11 were hospitalized for one or more clinical and/or surgical reasons (3 due to difficulties in controlling convulsive seizures, 5 due to swallowing disorders, insufficient weight gain and for gastrostomy, 9 due to upper or lower respiratory tract conditions, 2 due to urinary tract infections, 1 for a ventriculoperitoneal shunt placement and 1 due to a gastrostomy site infection).

The medications used during the Holter monitoring were as follows: 10 patients used anticonvulsants, including valproic acid, clobazam, topiramate, phenytoin and vigabatrin; 8 patients used oxybutynin; 2 used amoxicillin with clavulanate; 5 used ranitidine; 4 used beta-sympathomimetics and 1 used oral corticosteroids. The 4 patients hospitalized for Holter monitoring received only multivitamins. The average sleep duration during the 24 hours was 11 hours and 40 minutes. No patient was excluded from the study as a consequence of less than 18 hours of Holter clean trace.

There was no record of pauses, junctional rhythm, supraventricular or ventricular extrasystoles, blockages or
QT interval alterations. The mean HR was higher among infants with severe microcephaly and CZS, and HRV measurements had lower median values in the groups with CZS and severe microcephaly. No difference was detected between the groups with and without positive PCR and with and without fetal distress and among the groups of born SGA, PT or not. The data show that in the CZS and microcephaly groups, there was a significant difference in SDNN values ( $p<0.05$ in both groups) and $R-R$ values ( $p<0.10$ in the group with microcephaly and $\mathrm{p}<0.05$ in the group with CZS). The mean HR, pNN50 and rMSSD measurements showed significantly different values ( $p$-value $<0.10$ ) in the groups with and without microcephaly and CZS (Table 1).

\section{DISCUSSION}

The evaluation of HRV as an instrument to obtain information about neurodevelopment in children with some risk of cerebral damage in the perinatal period was proposed by Bjelakovic et al. ${ }^{19}$, who studied 35 infants aged between 2 and 6 months and diagnosed with mild to severe central coordination disorders and 37 healthy controls. Patients in the group with coordination disorders were followed up for 18 months and reevaluated regarding neurological changes (late diagnosis of cerebral palsy). The study suggested that SDNN values $<48$ milliseconds (ms) were predictive of worse neurological outcome and diagnosis of cerebral palsy at 18 months. In our sample, four patients with CZS presented SDNN less than $48 \mathrm{~ms}$ and there was a significant difference between the SDNN values for the groups with and without CZS; the values for the group with CZS were lower than those of the group without CZS. This finding suggests that the evaluation of HRV in young infants with vertical exposure to ZIKV and born without CZS can indicate who among these patients will develop worsening symptoms of delayed neuropsychomotor development during the first year of life. In addition, Galland et al. ${ }^{1}$ studied the HRV of 23 SGA newborns with idiopathic delayed intrauterine growth at 1 and 3 months, suggesting that the predominance of the sympathetic ANS observed by lower HRV in SGA newborns may be related to an increased risk of hypertension, diabetes mellitus, and other cardiovascular diseases in adulthood. In our sample, only two patients were born SGA, making the p-value analysis difficult even when significant differences between the SGA and non-SGA groups exists. The finding of lower SDNN in infants with CZS who participated in our study draws attention to other comorbidities that may develop during the life of these patients and to the need for a follow-up and measures to prevent cardiovascular diseases. 
Table 1 - Median values of the parameters of heart rate variability in 15 infants with vertical exposure to ZIKV according to the subgroup.

\begin{tabular}{|c|c|c|c|c|c|c|c|}
\hline \multirow{3}{*}{ Variables } & \multirow{3}{*}{ Categories } & \multirow{3}{*}{$\mathrm{n}$} & \multicolumn{5}{|c|}{ Parameters } \\
\hline & & & $\mathrm{HRm}($ beat/min) & pNN50(\%) & $\mathrm{rMSSD}(\mathrm{m} / \mathrm{s})$ & $\mathrm{R}-\mathrm{R}(\mathrm{s})$ & $\operatorname{SDNN}(\mathrm{m} / \mathrm{s})$ \\
\hline & & & $\begin{array}{c}\text { Median } \\
\text { (Min-Max) }\end{array}$ & $\begin{array}{c}\text { Median } \\
\text { (Min-Max) }\end{array}$ & $\begin{array}{c}\text { Median } \\
\text { (Min-Max) }\end{array}$ & $\begin{array}{c}\text { Median } \\
\text { (Min-Max) }\end{array}$ & $\begin{array}{c}\text { Median } \\
(\text { Min-Max) }\end{array}$ \\
\hline \multirow{3}{*}{ Sex } & Female & 6 & $\begin{array}{c}122.5 \\
(103-154)\end{array}$ & $\begin{array}{c}15.5 \\
(0-25)\end{array}$ & $\begin{array}{l}37.0 \\
(9-49)\end{array}$ & $\begin{array}{c}480.7 \\
(381.1-604.5)\end{array}$ & $\begin{array}{c}67.5 \\
(45-83)\end{array}$ \\
\hline & Male & 9 & $\begin{array}{c}128.0 \\
(102-174)\end{array}$ & $\begin{array}{c}5.0 \\
(2-23) \\
\end{array}$ & $\begin{array}{c}24.0 \\
(17-47) \\
\end{array}$ & $\begin{array}{c}465.1 \\
(357.1-593.3) \\
\end{array}$ & $\begin{array}{c}65.0 \\
(32-94) \\
\end{array}$ \\
\hline & $p$-value & & 0.7230 & 0.4780 & 0.4420 & 10.000 & 0.9550 \\
\hline \multirow{3}{*}{ Fetal distress } & No & 13 & $\begin{array}{c}128.0 \\
(102-174)\end{array}$ & $\begin{array}{c}5.0 \\
(0-25)\end{array}$ & $\begin{array}{l}25.0 \\
(9-49)\end{array}$ & $\begin{array}{c}465.1 \\
(357.1-604.5)\end{array}$ & $\begin{array}{c}65.0 \\
(37-94)\end{array}$ \\
\hline & Yes & 2 & $\begin{array}{c}125.5 \\
(114-137) \\
\end{array}$ & $\begin{array}{c}10.5 \\
(2-19) \\
\end{array}$ & $\begin{array}{c}30.5 \\
(17-44) \\
\end{array}$ & $\begin{array}{c}475.6 \\
(430-521.2) \\
\end{array}$ & $\begin{array}{c}57.5 \\
(32-83) \\
\end{array}$ \\
\hline & $\mathrm{p}$-value & & 0.7990 & 0.8650 & 0.9320 & 10.000 & 0.8000 \\
\hline \multirow{3}{*}{ PCR-NB } & No & 7 & $\begin{array}{c}139.0 \\
(109-174)\end{array}$ & $\begin{array}{c}5.0 \\
(0-18)\end{array}$ & $\begin{array}{l}24.0 \\
(9-39)\end{array}$ & $\begin{array}{c}455.8 \\
(357.1-560.1)\end{array}$ & $\begin{array}{c}58.0 \\
(37-79)\end{array}$ \\
\hline & Yes & 8 & $\begin{array}{c}116.0 \\
(102-137) \\
\end{array}$ & $\begin{array}{c}14.5 \\
(2-25) \\
\end{array}$ & $\begin{array}{c}37.0 \\
(17-49) \\
\end{array}$ & $\begin{array}{c}510.6 \\
(425.1-539.2) \\
\end{array}$ & $\begin{array}{c}76.0 \\
(32-94)\end{array}$ \\
\hline & $p$-value & & 0.0558 & 0.2450 & 0.2020 & 0.1520 & 0.1210 \\
\hline \multirow{3}{*}{ SGA } & No & 13 & $\begin{array}{c}123.0 \\
(102-174)\end{array}$ & $\begin{array}{c}10.0 \\
(0-25)\end{array}$ & $\begin{array}{l}31.0 \\
(9-49)\end{array}$ & $\begin{array}{c}488.7 \\
(357.1-604.5)\end{array}$ & $\begin{array}{c}74.0 \\
(32-94)\end{array}$ \\
\hline & Yes & 2 & $\begin{array}{c}132.5 \\
(109-154) \\
\end{array}$ & $\begin{array}{c}2.5 \\
(2-12) \\
\end{array}$ & $\begin{array}{c}19.0 \\
(17-31) \\
\end{array}$ & $\begin{array}{c}445.1 \\
(381.1-560.1) \\
\end{array}$ & $\begin{array}{c}62.5 \\
(45-65) \\
\end{array}$ \\
\hline & $\mathrm{p}$-value & & 0.6010 & 0.1490 & 0.1500 & 0.4890 & 0.3430 \\
\hline \multirow{3}{*}{ PT } & No & 14 & $\begin{array}{c}128.0 \\
(102-174)\end{array}$ & $\begin{array}{c}5.0 \\
(0-23)\end{array}$ & $\begin{array}{l}24.5 \\
(9-47)\end{array}$ & $\begin{array}{c}464.3 \\
(357.1-593.4)\end{array}$ & $\begin{array}{c}64.5 \\
(32-94)\end{array}$ \\
\hline & Yes & 1 & $\begin{array}{c}103.0 \\
(103-103) \\
\end{array}$ & $\begin{array}{c}25.0 \\
(25-25) \\
\end{array}$ & $\begin{array}{c}49.0 \\
(49-49) \\
\end{array}$ & $\begin{array}{c}604.5 \\
(604.5-604.5) \\
\end{array}$ & $\begin{array}{c}78.0 \\
(78-78) \\
\end{array}$ \\
\hline & $\mathrm{p}$-value & & 0.1640 & 0.1040 & 0.1040 & 0.1330 & 0.6670 \\
\hline \multirow{3}{*}{ Severe Microcephaly } & No & 7 & $\begin{array}{c}115.0 \\
(102-141)\end{array}$ & $\begin{array}{c}19.0 \\
(0-25)\end{array}$ & $\begin{array}{l}43.0 \\
(9-49)\end{array}$ & $\begin{array}{c}515.7 \\
(436.2-604.5)\end{array}$ & $\begin{array}{c}78.0 \\
(46-94)\end{array}$ \\
\hline & Yes & 8 & $\begin{array}{c}137.0 \\
(109-174) \\
\end{array}$ & $\begin{array}{c}3.5 \\
(2-18) \\
\end{array}$ & $\begin{array}{c}22.0 \\
(17-39) \\
\end{array}$ & $\begin{array}{c}446.7 \\
(357.1-560.1) \\
\end{array}$ & $\begin{array}{c}59.5 \\
(32-79) \\
\end{array}$ \\
\hline & $\mathrm{p}$-value & & 0.0558 & 0.0716 & 0.0818 & 0.0541 & 0.0205 \\
\hline \multirow{3}{*}{ CZS } & No & 3 & $\begin{array}{c}114.0 \\
(103-115)\end{array}$ & $\begin{array}{c}19.0 \\
(10-25)\end{array}$ & $\begin{array}{c}44.0 \\
(31-49)\end{array}$ & $\begin{array}{c}521.2 \\
(515.7-604.5)\end{array}$ & $\begin{array}{c}83.0 \\
(78-86)\end{array}$ \\
\hline & Yes & 12 & $\begin{array}{c}139.5 \\
(102-174) \\
\end{array}$ & $\begin{array}{c}4.5 \\
(0-23) \\
\end{array}$ & $\begin{array}{l}24.0 \\
(9-47) \\
\end{array}$ & $\begin{array}{c}459.9 \\
(357.1-593.3) \\
\end{array}$ & $\begin{array}{c}62.5 \\
(32-94) \\
\end{array}$ \\
\hline & $\mathrm{p}$-value & & 0.0601 & 0.0819 & 0.0507 & 0.0484 & 0.0484 \\
\hline
\end{tabular}

$\mathrm{HRm}=$ heart rate in beats per minute; pNN50 = average number of times per hour where R-R intervals exceed 50 milliseconds; rMMSD = square root of successive differences between normal heart beats; $R-R=$ interval in milliseconds between 2 consecutive $\mathrm{R}$ waves; SDNN = standard deviation of R-R intervals during the entire Holter "clean trace" time; SGA = small for gestational age; PT: preterm birth; PCR-NB: PCR of the newborn.

The study of HRV has also been used to assess the likelihood of the sudden infant death syndrome (SIDS), and it has been suggested that lower HRV and higher HR are associated with a higher probability of this outcome ${ }^{20}$. The follow-up of a cohort in the 1990s, composed of 6914 newborns and infants, among whom 16 died from SIDS and who performed 24-hour Holter monitoring in the first months of life, indicated an association of a higher HR and lower HRV with a higher probability of death by
SIDS $^{19}$. The evaluation of HRV in this study was performed through the analysis of the mean and standard deviation of the R-R. The study suggested that cardiac instabilities may cause potentially lethal arrhythmias, and during the period of increased ANS development, which coincides with the period of increased risk of SIDS, some children experience accelerated or asymmetrical development of the sympathetic arm of the ANS, placing them at a higher risk of SIDS. Our study showed a statistically significant difference 
between R-R values in patients with and without CZS (the CZS group showed a lower R-R value), but participants were older in our sample than in the aforementioned cohort, making it difficult to compare the absolute values.

During monitoring, all the patients, except for the four patients hospitalized only for Holter monitoring, were taking medications (anticonvulsants, anticholinergics, histamine receptor antagonists, corticosteroids, beta-sympathomimetics and antibiotics). Baysal-Kirac et al. ${ }^{21}$ suggested that patients with drug-resistant epilepsy present with significantly lower HRV measures, which may increase the risk of sudden cardiac death. Increased heart rate and diminished HRV measures may constitute one of the possible mechanisms underlying sudden unexpected deaths in epilepsy and should be diagnosed in patients with epilepsy. We found no other studies on HRV and the other medications used by our patients. The patients in our sample who were not using anticonvulsants, were in the group without CZS and had higher HRV than those in the CZS group.

The results of this study should be interpreted with caution in light of its limitations, such as the small sample size, and its strengths, which are the novelty of the information and the importance of proposing a sentinel event for the prediction of worse neurological evolution and the possibility of cardiovascular comorbidities in patients with prenatal exposure to ZIKV. Due to the absence of normal HRV values for patients in the age group of our study, we chose to describe the values in this group of infants with vertical exposure to ZIKV and compare the values among subgroups of the sample.

This study is unprecedented in a population of infants in this age group and with vertical exposure to ZIKV. Given the seasonality of arboviruses infections, we cannot predict when it will be possible to repeat the study in a larger sample. The findings in the 24-hour Holter monitoring suggest that infants with in utero exposure to ZIKV and severe CZS are at higher risk of SIDS. In addition, the results call attention to the development of possible comorbidities, such as cardiovascular diseases, that may present in the medium/ long term and require early prevention measures.

\section{ACKNOWLEDGMENTS}

SRLP was supported by Conselho Nacional de Desenvolvimento Científico e Tecnológico (CNPq), grants No 440846/2016-1 and 310765/2016-1, by Coordenação de Aperfeiçoamento de Pessoal de Nível Superior (CAPES), grant $\mathrm{N}^{\circ} 88881130793 / 2016-01$, and by Universidade Estácio de Sá - Produtividade em Pesquisa. LMLS was supported by CAPES grant $\mathrm{N}^{\circ}$ 88887.162025/2017-00. RVG and TMR had a scholarship ITI-A from CNPq.

\section{REFERENCES}

1. Galland BC, Taylor BJ, Bolton DP, Sayers RM. Heart rate variability and cardiac reflexes in small for gestational age infants. J Appl Physiol. 2006;100:933-9.

2. Rakow A, Katz-Salamon M, Ericson M, Edner A, Vanpée M. Decreased heart rate variability in children born with low birth weight. Pediatr Res. 2013;74:339-43.

3. Franciosi S, Perry FK, Roston TM, Armstrong KR, Claydon VE, Sanatani S. The role of the autonomic nervous system in arrhythmias and sudden cardiac death. Auton Neurosci. 2017;205:1-11.

4. Shaffer F, McCraty R, Zerr CL. A healthy heart is not a metronome: an integrative review of the heart's anatomy and heart rate variability. Front Psychol. 2014;5:1040.

5. Massin M, von Bernuth G. Normal ranges of heart rate variability during infancy and childhood. Pediatr Cardiol. 1997;18:297302.

6. Vanderlei LC, Pastre CM, Hoshi RA, Carvalho TD, Godoy MF. Basic notions of heart rate variability and its clinical applicability. Rev Bras Cir Cardiovasc. 2009;24:205-17.

7. Schechtman VL, Harper RM, Kluge KA, Wilson AJ, Hoffman HJ, Southall DP. Cardiac and respiratory patterns in normal infant victims of sudden infant death syndrome. Sleep. 1988;11:41324

8. Fox N, Porges $\mathrm{S}$. The relation between neonatal period patterns and development outcome. Child Dev. 1985;56:28-37.

9. Doussard-Roosevelt J, Porges S, Scanlon J, Alemi B, Scanlon K. Vagal regulation of heart rate in the prediction of developmental outcome for very low birth weight preterm infants. Chil Dev. 1997;68:173-86.

10. Moore CA, Staples JE, Dobyns WB, Pessoa A, Ventura CV, Fonseca EB, et al. Characterizing the pattern of anomalies in congenital Zika syndrome for pediatric clinicians. JAMA Pediatr. 2017;171:288-95.

11. Meneses JD, Ishigami AC, Mello LM, Albuquerque LL, Brito CA, Cordeiro MT, et al. Lessons learned at the epicenter of Brazil's congenital Zika epidemic: evidence from 87 confirmed cases. Clin Infect Dis. 2017;64:1302-8.

12. Orofino DH, Passos SR, Oliveira RV, Farias CV, Leite MF, Pone $\mathrm{SM}$, et al. Cardiac findings in infants with in utero exposure to Zika virus: a cross sectional study. PLoS Negl Trop Dis. 2018;12:e006362.

13. Lanciotti RS, Kosoy OL, Laven JJ, Velez JO, Lambert AJ, Johnson AJ, et al. Genetic and serologic properties of Zika virus associated with an epidemic, Yap State, Micronesia, 2007. Emerg Infect Dis. 2008;14:1232-9.

14. Rabe IB, Staples JE, Villanueva J, Hummel KB, Johnson JA, Rose L, et al. Interim guidance for interpretation of Zika virus antibody test results. MMWR Morb Mortal Wkly Rep. 2016;65:543-6. 
15. American Academy of Pediatrics Committee on Fetus and Newborn, American College of Obstetricians and Gynecologists Committee on Obstetric Practice. The Apgar Score. Pediatrics. 2015;136:819-22.

16. Lawn JE, Gravett MG, Nunes TM, Rubens CE, Stanton C. Global report on preterm birth and stillbirth (1 of 7): definitions, description of the burden and opportunities to improve data. BMC Pregnancy Childbirth. 2010;10 Suppl 1:S1.

17. Stirnemann J, Villar J, Salomon LJ, Ohuma E, Ruyan P, Altman DG, et al. International estimate fetal weight standards of the INTERGROWTH-2 $1^{\text {st }}$ Project. Ultrasound Obstet Gynecol. 2017;49:478-86

18. Task Force of the European Society of Cardiology and the North American Society of Pacing and Eletrophysiology. Heart rate variability: standards of measurement, physiological interpretation and clinical use. Circulation. 1996;93:1043-65.

19. Bjelakovic B, Ilic S, Dimitrijevic L, Milovanovic B, Kostic G, Bjelakovic L, et al. Heart rate variability in infants with central coordination disturbance. Early Hum Dev. 2010;86:77-81.

20. Schechtman VL, Raetz SL, Harper RK, Garfinkel A, Wilson AJ, Southall DP, et al. Dynamic analysis of cardiac R-R intervals in normal infants and in infants who subsequently succumbed to the sudden infant death syndrome. Pediatr Res. 1992;31:60612 .

21. Baysal-Kirac L, Serbest NG, Şahin E, Dede HO, Gurses C, Gokyigit A, et al. Analysis of heart rate variability and risk factors for SUDEP in patients with drug-resistant epilepsy. Epilepsy Behav. 2017;71:60-4. 